\title{
Existence of Solutions for Integrodifferential Equations of Fractional Order with Antiperiodic Boundary Conditions
}

\begin{abstract}
Ahmed Alsaedi
Department of Mathematics, Faculty of Science, King Abdulaziz University, P.O. Box 80203, Jeddah 21589, Saudi Arabia

Correspondence should be addressed to Ahmed Alsaedi, aalsaedi@hotmail.com

Received 13 August 2009; Accepted 12 November 2009

Academic Editor: Juan Jose Nieto

Copyright (C) 2009 Ahmed Alsaedi. This is an open access article distributed under the Creative Commons Attribution License, which permits unrestricted use, distribution, and reproduction in any medium, provided the original work is properly cited.

We discuss the existence of solutions for a nonlinear antiperiodic boundary value problem of integrodifferential equations of fractional order $q \in(1,2]$. The contraction mapping principle and Krasnoselskii's fixed point theorem are applied to establish the results.
\end{abstract}

\section{Introduction}

Recently, the subject of fractional differential equations has emerged as an important area of investigation. Fractional differential equations arise in many engineering and scientific disciplines as the fractional derivatives describe numerous events and processes in the fields of physics, chemistry, aerodynamics, electrodynamics of complex medium, polymer rheology, and so forth. For some recent development on the subject, see [1-15] and the references therein.

Integrodifferential equations arise in many engineering and scientific disciplines, often as approximation to partial differential equations, which represent much of the continuum phenomena. Many forms of these equations are possible. For details, see [16-20] and the references therein.

Antiperiodic boundary value problems have recently received considerable attention as antiperiodic boundary conditions appear in numerous situations, for instance, see [21-25].

In this paper, we prove some existence and uniqueness results for the following antiperiodic fractional boundary value problem:

$$
\begin{array}{cl}
{ }^{c} D^{q} x(t)=f(t, x(t),(x x)(t)), & t \in[0, T], T>0,1<q \leq 2, \\
x(0)=-x(T), & x^{\prime}(0)=-x^{\prime}(T),
\end{array}
$$


where ${ }^{c} D^{q}$ denotes the Caputo fractional derivative of order $q, f:[0, T] \times X \times X \rightarrow X$, and for $\gamma:[0, T] \times[0, T] \rightarrow[0, \infty)$,

$$
(x x)(t)=\int_{0}^{t} \gamma(t, s) x(s) d s
$$

with $\gamma_{0}=\max \left\{\int_{0}^{t} \gamma(t, s) d s:(t, s) \in[0, T] \times[0, T]\right\}$. Here, $(X,\|\cdot\|)$ is a Banach space and $C=C([0, T], X)$ denotes the Banach space of all continuous functions from $[0, T] \rightarrow X$ endowed with a topology of uniform convergence with the norm denoted by $\|\cdot\|$.

\section{Preliminaries}

First of all, we recall some basic definitions [26-28].

Definition 2.1. For a function $g:[0, \infty) \rightarrow \mathbb{R}$, the Caputo derivative of fractional order $q$ is defined as

$$
{ }^{c} D^{q} g(t)=\frac{1}{\Gamma(n-q)} \int_{0}^{t}(t-s)^{n-q-1} g^{(n)}(s) d s, \quad n-1<q<n, n=[q]+1,
$$

where $[q]$ denotes the integer part of the real number $q$.

Definition 2.2. The Riemann-Liouville fractional integral of order $q$ is defined as

$$
I^{q} g(t)=\frac{1}{\Gamma(q)} \int_{0}^{t} \frac{g(s)}{(t-s)^{1-q}} d s, \quad q>0
$$

provided that the integral exists.

Definition 2.3. The Riemann-Liouville fractional derivative of order $q$ for a function $g(t)$ is defined by

$$
D^{q} g(t)=\frac{1}{\Gamma(n-q)}\left(\frac{d}{d t}\right)^{n} \int_{0}^{t} \frac{g(s)}{(t-s)^{q-n+1}} d s, \quad n=[q]+1
$$

provided that the right-hand side is pointwise defined on $(0, \infty)$.

Lemma 2.4 (see [8]). For $q>0$, the general solution of the fractional differential equation ${ }^{c} D^{q} x(t)=$ 0 is given by

$$
x(t)=c_{0}+c_{1} t+c_{2} t^{2}+\cdots+c_{n-1} t^{n-1},
$$

where $c_{i} \in \mathbb{R}, i=0,1,2, \ldots, n-1(n=[q]+1)$. 
In view of Lemma 2.4, it follows that

$$
I^{q c} D^{q} x(t)=x(t)+c_{0}+c_{1} t+c_{2} t^{2}+\cdots+c_{n-1} t^{n-1}
$$

for some $c_{i} \in \mathbb{R}, i=0,1,2, \ldots, n-1(n=[q]+1)$.

Now, we state a known result due to Krasnoselskii [29] which is needed to prove the existence of at least one solution of (1.1).

Theorem 2.5. Let $M$ be a closed convex and nonempty subset of a Banach space X. Let $A, B$ be the operators such that (i) $A x+B y \in M$ whenever $x, y \in M$; (ii) $A$ is compact and continuous; (iii) $B$ is a contraction mapping. Then there exists $z \in M$ such that $z=A z+B z$.

Lemma 2.6. For any $\omega \in C[0, T]$, the unique solution of the boundary value problem

$$
\begin{gathered}
{ }^{c} D^{q} x(t)=\omega(t), \quad 0<t<T, 1<q \leq 2, \\
x(0)=-x(T), \quad x^{\prime}(0)=-x^{\prime}(T)
\end{gathered}
$$

is given by

$$
x(t)=\int_{0}^{T} G(t, s) \omega(s) d s,
$$

where $G(t, s)$ is the Green's function given by

$$
G(t, s)= \begin{cases}\frac{(t-s)^{q-1}-(1 / 2)(T-s)^{q-1}}{\Gamma(q)}+\frac{(T-2 t)(T-s)^{q-2}}{4 \Gamma(q-1)}, & s \leq t, \\ -\frac{(T-s)^{q-1}}{2 \Gamma(q)}+\frac{(T-2 t)(T-s)^{q-2}}{4 \Gamma(q-1)}, & t \leq s .\end{cases}
$$

Proof. Using (2.5), for some constants $b_{1}, b_{2} \in \mathbb{R}$, we have

$$
x(t)=I^{q} \omega(t)-b_{1}-b_{2} t=\int_{0}^{t} \frac{(t-s)^{q-1}}{\Gamma(q)} \omega(s) d s-b_{1}-b_{2} t .
$$

In view of the relations ${ }^{c} D^{q} I^{q} x(t)=x(t)$ and $I^{q} I^{p} x(t)=I^{q+p} x(t)$ for $q, p>0, x \in L(0,1)$, we obtain

$$
x^{\prime}(t)=\int_{0}^{t} \frac{(t-s)^{q-2}}{\Gamma(q-1)} \omega(s) d s-b_{2} .
$$


Applying the boundary conditions $x(0)=-x(T), x^{\prime}(0)=-x^{\prime}(T)$, we find that

$$
\begin{gathered}
b_{1}=\frac{1}{2 \Gamma(q)} \int_{0}^{T}(T-s)^{q-1} \omega(s) d s-\frac{T}{4 \Gamma(q-1)} \int_{0}^{T}(T-s)^{q-2} \omega(s) d s, \\
b_{2}=\frac{1}{2 \Gamma(q-1)} \int_{0}^{T}(T-s)^{q-2} \omega(s) d s .
\end{gathered}
$$

Thus, the unique solution of (2.6) is

$$
\begin{aligned}
x(t)= & \int_{0}^{t} \frac{(t-s)^{q-1}}{\Gamma(q)} \omega(s) d s-\frac{1}{2} \int_{0}^{T} \frac{(T-s)^{q-1}}{\Gamma(q)} \omega(s) d s \\
& +\frac{1}{4}(T-2 t) \int_{0}^{T} \frac{(T-s)^{q-2}}{\Gamma(q-1)} \omega(s) d s \\
= & \int_{0}^{T} G(t, s) \omega(s) d s,
\end{aligned}
$$

where $G(t, s)$ is given by (2.8). This completes the proof.

\section{Main Results}

To prove the main results, we need the following assumptions:

$\left(A_{1}\right)\|f(t, x(t),(\chi x)(t))-f(t, y(t),(x y)(t))\| \leq L_{1}\|x-y\|+\bar{L}_{1}\|x x-x y\|$, for all $t \in[0, T]$, $x, y \in X$;

$\left(A_{2}\right)\|f(t, x(t),(\chi x)(t))\| \leq \mu(t)$, for all $\left(t, x, \chi^{x}\right) \in[0, T] \times X \times X$ and $\mu \in L^{1}\left([0, T], R^{+}\right)$.

Theorem 3.1. Let $f:[0, T] \times X \times X \rightarrow X$ be a jointly continuous function satisfying the assumption $\left(A_{1}\right)$ with $\left(L_{1}+\gamma_{0} \bar{L}_{1}\right) \leq \Gamma(q+1) / T^{q}(3+q / 2)$. Then the antiperiodic boundary value problem (1.1) has a unique solution.

Proof. Define $\Theta: C \rightarrow C$ by

$$
\begin{aligned}
(\Theta x)(t)= & \int_{0}^{t} \frac{(t-s)^{q-1}}{\Gamma(q)} f\left(s, x(s),\left(\chi^{x}\right)(s)\right) d s \\
& -\frac{1}{2} \int_{0}^{T} \frac{(T-s)^{q-1}}{\Gamma(q)} f(s, x(s),(\chi x)(s)) d s \\
& +\frac{1}{4}(T-2 t) \int_{0}^{T} \frac{(T-s)^{q-2}}{\Gamma(q-1)} f(s, x(s),(x x)(s)) d s, \quad t \in[0, T]
\end{aligned}
$$


Setting $\sup _{t \in[0, T]}\|f(t, 0,0)\|=M$ and choosing $r \geq\left(M T^{q} / \Gamma(q+1)\right)(3+q / 2)$, we show that $\Theta B_{r} \subset B_{r}$, where $B_{r}=\{x \in C:\|x\| \leq r\}$. For $x \in B_{r}$, we have

$$
\begin{aligned}
& \|(\Theta x)(t)\| \leq \int_{0}^{t} \frac{(t-s)^{q-1}}{\Gamma(q)}\|f(s, x(s),(x x)(s))\| d s \\
& +\frac{1}{2} \int_{0}^{T} \frac{(T-s)^{q-1}}{\Gamma(q)}\|f(s, x(s),(x x)(s))\| d s \\
& +\frac{1}{4}|T-2 t| \int_{0}^{T} \frac{(T-s)^{q-2}}{\Gamma(q-1)}\|f(s, x(s),(x x)(s))\| d s \\
& \leq \int_{0}^{t} \frac{(t-s)^{q-1}}{\Gamma(q)}[\|f(s, x(s),(x x)(s))-f(s, 0,0)\|+\|f(s, 0,0)\|] d s \\
& +\frac{1}{2} \int_{0}^{T} \frac{(T-s)^{q-1}}{\Gamma(q)}[\|f(s, x(s),(x x)(s))-f(s, 0,0)\|+\|f(s, 0,0)\|] d s \\
& +\frac{1}{4}|T-2 t| \int_{0}^{T} \frac{(T-s)^{q-2}}{\Gamma(q-1)}[\|f(s, x(s),(x x)(s))-f(s, 0,0)\|+\|f(s, 0,0)\|] d s \\
& \leq\left(\left(L_{1}+\gamma_{0} \bar{L}_{1}\right) r+M\right)\left[\frac{1}{\Gamma(q)} \int_{0}^{t}(t-s)^{q-1} d s+\frac{1}{2 \Gamma(q)} \int_{0}^{T}(T-s)^{q-1} d s\right. \\
& \left.+\frac{1}{4 \Gamma(q-1)}|T-2 t| \int_{0}^{T}(T-s)^{q-2} d s\right] \\
& \leq\left(\left(L_{1}+\gamma_{0} \bar{L}_{1}\right) r+M\right)\left[\frac{T^{q}}{2 \Gamma(q+1)}\left(3+\frac{q}{2}\right)\right] \leq r .
\end{aligned}
$$

Now, for $x, y \in C$ and for each $t \in[0, T]$, we obtain

$$
\begin{aligned}
\|(\Theta x)(t)-(\Theta y)(t)\| \leq & \int_{0}^{t} \frac{(t-s)^{q-1}}{\Gamma(q)}\|f(s, x(s),(x x)(s))-f(s, y(s),(x y)(s))\| d s \\
& +\frac{1}{2} \int_{0}^{T} \frac{(T-s)^{q-1}}{\Gamma(q)}\|f(s, x(s),(x x)(s))-f(s, y(s),(x y)(s))\| d s \\
& +\frac{1}{4}|T-2 t| \int_{0}^{T} \frac{(T-s)^{q-2}}{\Gamma(q-1)}\|f(s, x(s),(x x)(s))-f(s, y(s),(x y)(s))\| d s
\end{aligned}
$$




$$
\begin{aligned}
& \leq\left(L_{1}+\gamma_{0} \bar{L}_{1}\right)\|x-y\|\left[\frac{1}{\Gamma(q)} \int_{0}^{t}(t-s)^{q-1} d s+\frac{1}{2 \Gamma(q)} \int_{0}^{T}(T-s)^{q-1} d s\right. \\
& \left.\quad+\frac{1}{4 \Gamma(q-1)}|T-2 t| \int_{0}^{T}(T-s)^{q-2} d s\right] \\
& \leq \frac{\left(L_{1}+\gamma_{0} \bar{L}_{1}\right) T^{q}}{2 \Gamma(q+1)}\left(3+\frac{q}{2}\right)\|x-y\| \\
& \leq \Lambda_{L_{1}, \bar{L}_{1}, \gamma_{0}, T, q}\|x-y\|,
\end{aligned}
$$

where $\Lambda_{L_{1}, \bar{L}_{1}, \gamma_{0}, T, q}=\left(\left(L_{1}+\gamma_{0} \bar{L}_{1}\right) T^{q} / 2 \Gamma(q+1)\right)(3+q / 2)$, which depends only on the parameters involved in the problem. As $\Lambda_{L_{1}, \bar{L}_{1}, \gamma_{0}, T, q}<1$, therefore $\Theta$ is a contraction. Thus, the conclusion of the theorem follows by the contraction mapping principle (Banach fixed point theorem).

Theorem 3.2. Let $f:[0, T] \times X \times X \rightarrow X$ be a jointly continuous function mapping bounded subsets of $[0,1] \times X \times X$ into relatively compact subsets of $X$, and the assumptions $\left(A_{1}\right)-\left(A_{2}\right)$ hold with $\left(\left(L_{1}+\gamma_{0} \bar{L}_{1}\right) T^{q} / 2 \Gamma(q+1)\right)(1+q / 2)<1$. Then the antiperiodic boundary value problem (1.1) has at least one solution on $[0, T]$.

Proof. Let us fix

$$
r \geq \frac{\|\mu\|_{L^{1}} T^{q}}{2 \Gamma(q+1)}\left(3+\frac{q}{2}\right)
$$

and consider $B_{r}=\{x \in C:\|x\| \leq r\}$. We define the operators $\Phi$ and $\Psi$ on $B_{r}$ as

$$
\begin{aligned}
(\Phi x)(t)= & \frac{1}{\Gamma(q)} \int_{0}^{t}(t-s)^{q-1} f\left(s, x(s),\left(\chi^{x}\right)(s)\right) d s \\
(\Psi x)(t)= & -\frac{1}{2} \int_{0}^{T} \frac{(T-s)^{q-1}}{\Gamma(q)} f\left(s, x(s),\left(\chi^{x}\right)(s)\right) d s \\
& +\frac{1}{4}(T-2 t) \int_{0}^{T} \frac{(T-s)^{q-2}}{\Gamma(q-1)} f\left(s, x(s),\left(\chi^{x}\right)(s)\right) d s
\end{aligned}
$$

For $x, y \in B_{r}$, we find that

$$
\|\Phi x+\Psi y\| \leq \frac{\|\mu\|_{L_{1}} T^{q}}{2 \Gamma(q+1)}\left(3+\frac{q}{2}\right) \leq r
$$


Thus, $\Phi x+\Psi y \in B_{r}$. It follows from the assumption $\left(A_{1}\right)$ that $\Psi$ is a contraction mapping for $\left(\left(L_{1}+\gamma_{0} \bar{L}_{1}\right) T^{q} / 2 \Gamma(q+1)\right)(1+q / 2)<1$. Continuity of $f$ implies that the operator $\Phi$ is continuous. Also, $\Phi$ is uniformly bounded on $B_{r}$ as

$$
\|\Phi x\| \leq \frac{\|\mu\|_{L^{1}} T^{q}}{\Gamma(q+1)} .
$$

Now we prove the compactness of the operator $\Phi$. In view of $\left(A_{1}\right)$, we define $\sup _{(t, x, x x) \in \Omega}\|f(t, x,(\chi x))\|=f_{\max }, \Omega=[0, T] \times B_{r} \times B_{r}$, and consequently we have

$$
\begin{aligned}
\left\|(\Phi x)\left(t_{1}\right)-(\Phi x)\left(t_{2}\right)\right\|= & \| \frac{1}{\Gamma(q)} \int_{0}^{t_{1}}\left[\left(t_{2}-s\right)^{q-1}-\left(t_{1}-s\right)^{q-1}\right] f(s, x(s),(x x)(s)) d s \\
& \quad+\int_{t_{1}}^{t_{2}}\left(t_{2}-s\right)^{q-1} f(s, x(s),(x x)(s)) d s \| \\
\leq & \frac{f_{\max }}{\Gamma(q+1)}\left|2\left(t_{2}-t_{1}\right)^{q}+t_{1}^{q}-t_{2}^{q}\right|
\end{aligned}
$$

which is independent of $x$. So $\Phi$ is relatively compact on $B_{r}$. Hence, by Arzela Ascoli theorem, $\Phi$ is compact on $B_{r}$. Thus all the assumptions of Theorem 2.5 are satisfied and the conclusion of Theorem 2.5 implies that the antiperiodic boundary value problem (1.1) has at least one solution on $[0, T]$.

Example 3.3. Consider the following antiperiodic boundary value problem:

$$
\begin{gathered}
{ }^{c} D^{q} x(t)=\frac{1}{(t+4)^{2}} \frac{\|x\|}{1+\|x\|}+\int_{0}^{t} \frac{e^{-(s-t)}}{16} x(s) d s, \quad t \in[0,1], 1<q \leq 2, \\
x(0)=-x(1), \quad x^{\prime}(0)=-x^{\prime}(1) .
\end{gathered}
$$

Here, $f(t, x)=\left(1 /(t+4)^{2}\right)(\|x\| /(1+\|x\|)), \gamma(t, s)=e^{-(s-t)} / 16, T=1$. Clearly,

$$
\|f(t, x, x x)-f(t, y, x y)\| \leq \frac{1}{16}(\|x-y\|+\|x x-x y\|) .
$$

So $\left(A_{1}\right)$ is satisfied with $L_{1}=\bar{L}_{1}=1 / 16$. Further

$$
\frac{\left(L_{1}+\gamma_{0} \bar{L}_{1}\right) T^{q}}{\Gamma(q+1)}\left(3+\frac{q}{2}\right)<1 \Longleftrightarrow \frac{(3+q / 2)}{\Gamma(q+1)}<\frac{16}{e} .
$$

Thus, by Theorem 3.1, the boundary value problem (3.9) has a unique solution on $[0,1]$. 


\section{References}

[1] V. Daftardar-Gejji and S. Bhalekar, "Boundary value problems for multi-term fractional differential equations," Journal of Mathematical Analysis and Applications, vol. 345, no. 2, pp. 754-765, 2008.

[2] A. Yang and W. Ge, "Positive solutions for boundary value problems of N-dimension nonlinear fractional differential system," Boundary Value Problems, vol. 2008, Article ID 437453, 15 pages, 2008.

[3] S. Z. Rida, H. M. El-Sherbiny, and A. A. M. Arafa, "On the solution of the fractional nonlinear Schrödinger equation," Physics Letters A, vol. 372, no. 5, pp. 553-558, 2008.

[4] B. Ahmad, "Some existence results for boundary value problems of fractional semilinear evolution equations," Electronic Journal of Qualitative Theory of Differential Equations, no. 28, pp. 1-7, 2009.

[5] B. Ahmad and J. J. Nieto, "Existence of solutions for nonlocal boundary value problems of higherorder nonlinear fractional differential equations," Abstract and Applied Analysis, vol. 2009, Article ID 494720, 9 pages, 2009.

[6] B. Ahmad and S. Sivasundaram, "Existence results for nonlinear impulsive hybrid boundary value problems involving fractional differential equations," Nonlinear Analysis: Hybrid Systems, vol. 3, no. 3, pp. 251-258, 2009.

[7] Y.-K. Chang and J. J. Nieto, "Some new existence results for fractional differential inclusions with boundary conditions," Mathematical and Computer Modelling, vol. 49, no. 3-4, pp. 605-609, 2009.

[8] V. Lakshmikantham, S. Leela, and J. V. Devi, Theory of Fractional Dynamic Systems, Cambridge Academic, Cambridge, UK, 2009.

[9] X. Su and S. Zhang, "Solutions to boundary-value problems for nonlinear differential equations of fractional order," Electronic Journal of Differential Equations, vol. 2009, no. 26, p. 115, 2009.

[10] V. Gafiychuk, B. Datsko, V. Meleshko, and D. Blackmore, "Analysis of the solutions of coupled nonlinear fractional reaction-diffusion equations," Chaos, Solitons and Fractals, vol. 41, no. 3, pp. 10951104, 2009.

[11] M. Benchohra, A. Cabada, and D. Seba, "An existence result for nonlinear fractional differential equations on Banach spaces," Boundary Value Problems, vol. 2009, Article ID 628916, 11 pages, 2009.

[12] A. M. A. El-Sayed and H. H. G. Hashem, "Monotonic solutions of functional integral and differential equations of fractional order," Electronic Journal of Qualitative Theory of Differential Equations, no. 7, pp. 1-8, 2009.

[13] M. Benchohra and S. Hamani, "The method of upper and lower solutions and impulsive fractional differential inclusions," Nonlinear Analysis: Hybrid Systems, vol. 3, no. 4, pp. 433-440, 2009.

[14] A. Arara, M. Benchohra, N. Hamidi, and J. J. Nieto, "Fractional order differential equations on an unbounded domain," Nonlinear Analysis: Theory, Methods E Applications, vol. 72, no. 2, pp. 580-586, 2010.

[15] Y. Tian and A. Chen, "The existence of positive solution to three-point singular boundary value problem of fractional differential equation," Abstract and Applied Analysis, vol. 2009, Article ID 314656, 18 pages, 2009.

[16] B. Ahmad and B. S. Alghamdi, "Approximation of solutions of the nonlinear Duffing equation involving both integral and non-integral forcing terms with separated boundary conditions," Computer Physics Communications, vol. 179, no. 6, pp. 409-416, 2008.

[17] B. Ahmad, "On the existence of T-periodic solutions for Duffing type integro-differential equations with p-Laplacian," Lobachevskii Journal of Mathematics, vol. 29, no. 1, pp. 1-4, 2008.

[18] S. Mesloub, "On a mixed nonlinear one point boundary value problem for an integrodifferential equation," Boundary Value Problems, vol. 2008, Article ID 814947, 8 pages, 2008.

[19] B. Ahmad and J. J. Nieto, "Existence results for nonlinear boundary value problems of fractional integrodifferential equations with integral boundary conditions," Boundary Value Problems, vol. 2009, Article ID 708576, 11 pages, 2009.

[20] Y. K. Chang and J. J. Nieto, "Existence of solutions for impulsive neutral integrodifferential inclusions with nonlocal initial conditions via fractional operators," Numerical Functional Analysis and Optimization, vol. 30, no. 3, pp. 227-244, 2009.

[21] Y. Chen, J. J. Nieto, and D. O’Regan, "Anti-periodic solutions for fully nonlinear first-order differential equations," Mathematical and Computer Modelling, vol. 46, no. 9-10, pp. 1183-1190, 2007.

[22] B. Liu, "An anti-periodic LaSalle oscillation theorem for a class of functional differential equations," Journal of Computational and Applied Mathematics, vol. 223, no. 2, pp. 1081-1086, 2009.

[23] B. Ahmad and V. Otero-Espinar, "Existence of solutions for fractional differential inclusions with antiperiodic boundary conditions," Boundary Value Problems, vol. 2009, Article ID 625347, 11 pages, 2009. 
[24] B. Ahmad and J. J. Nieto, "Existence of solutions for anti-periodic boundary value problems involving fractional differential equations via Leray-Schauder degree theory," to appear in Topological Methods in Nonlinear Analysis.

[25] Y. Q. Chen, D. O’Regan, F. L. Wang, and S. L. Zhou, “Antiperiodic boundary value problems for finite dimensional differential systems," Boundary Value Problems, vol. 2009, Article ID 541435, 11 pages, 2009.

[26] I. Podlubny, Fractional Differential Equations, vol. 198 of Mathematics in Science and Engineering, Academic Press, San Diego, Calif, USA, 1999.

[27] R. Hilfer, Ed., Applications of Fractional Calculus in Physics, World Scientific, Singapore, 2000.

[28] A. A. Kilbas, H. M. Srivastava, and J. J. Trujillo, Theory and Applications of Fractional Differential Equations, vol. 204 of North-Holland Mathematics Studies, Elsevier, Amsterdam, The Netherlands, 2006.

[29] D. R. Smart, Fixed Point Theorems, Cambridge University Press, Cambridge, UK, 1980. 


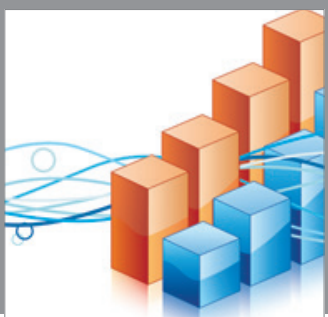

Advances in

Operations Research

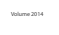

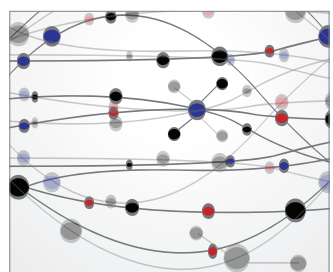

\section{The Scientific} World Journal
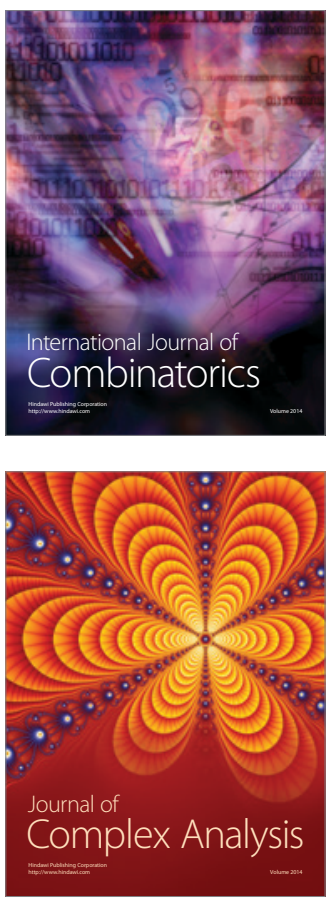

International Journal of

Mathematics and

Mathematical

Sciences
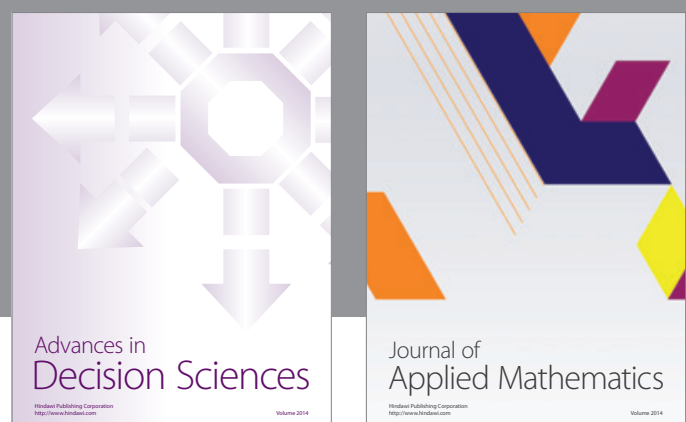

Journal of

Applied Mathematics
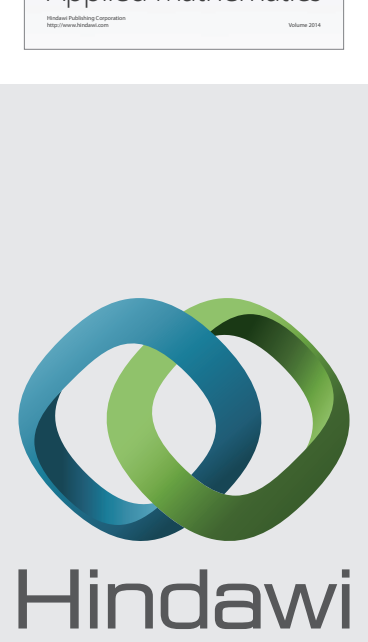

Submit your manuscripts at http://www.hindawi.com
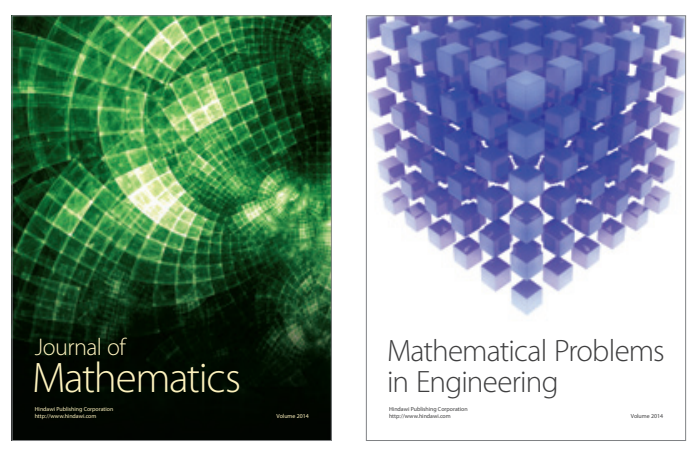

Mathematical Problems in Engineering
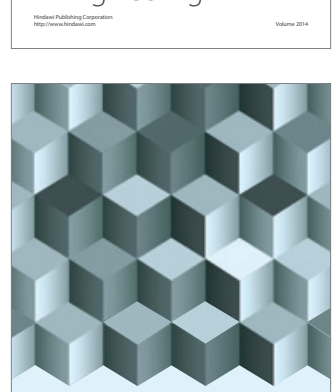

Journal of

Function Spaces
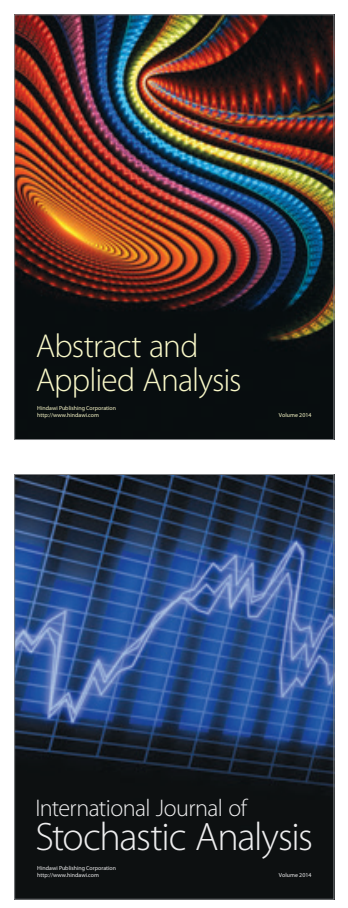

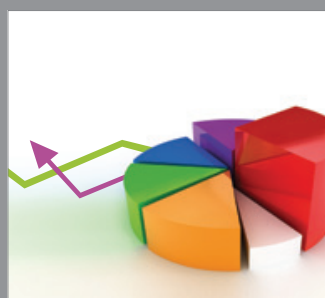

ournal of

Probability and Statistics

Promensencen
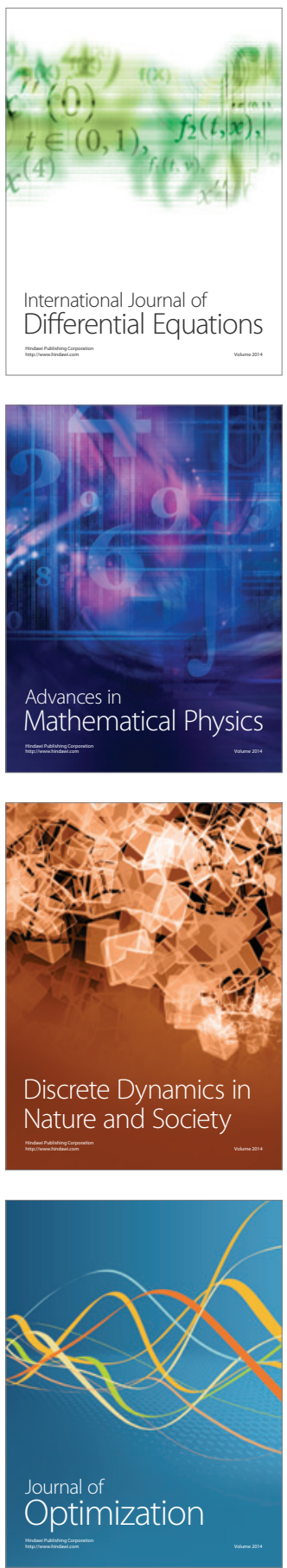